Investigations

\title{
Development of Genetic Constructions for Exctocytosis Control
}

\author{
${ }^{1}$ Irina Nikolaevna Dominova, ${ }^{2}$ Vitaly Anvarovich Kasymov, \\ ${ }^{3}$ Ekaterina Vladimirovna Silina, ${ }^{4}$ Victor Aleksandrovich Stupin, \\ ${ }^{5}$ Natalia Nikolaevna Shusharina and ${ }^{6}$ Maksim Vladimirovich Patrushev \\ 1,2,5,6 Immanuel Kant Baltic Federal University (IKBFU) Nevskogo Str., 14, Kaliningrad, 236041, Russia \\ ${ }^{3}$ I.M. Sechenov First Moscow State Medical University (Sechenov University) Trubetskaya Str, 8, Moscow, 119991, Russia \\ ${ }^{4}$ N.I. Pirogov Russian National Research Medical University (RNRMU) Ostrovityanova St., 1, Moscow, 117997, Russia
}

\author{
Article history \\ Received: 21-09-2017 \\ Revised: 25-11-2017 \\ Accepted: 29-01-2018 \\ Corresponding Author: \\ Irina Nikolaevna Dominova \\ Immanuel Kant Baltic Federal \\ University (IKBFU) Nevskogo \\ Str., 14, Kaliningrad, 236041, \\ Russia \\ E-mail: i-dominova@yandex.ru
}

\begin{abstract}
A fragment of the research project devoted to the development of genetic control of exocytosis is presented in the work, which can further ensure the success of targeted therapy of various diseases, including neurodegenerative ones. For the purpose of specific transfection of intracellular cascades in astrocytes in vivo, we have developed an experimental sample of the reagent kit. The latter represents lentiviral genetic construction LVV-GFAP-Case12. The results of testing of experimental samples of the reagent kit for specific transfection of astroglial cells are presented with the purpose of targeted control of intracellular cascades in vivo on acute slices of different parts of the brain in adult Wistar rats. We selected regions of interest with cells expressing calcium indicator Case 12 and responsible for ATP application by $>2$-fold amplification of fluorescence. We have developed instructions for using the reagent kit for specific transfection of astroglial cells with the purpose of targeted control of intracellular cascades.
\end{abstract}

Keywords: Astrocytes, Astroglia, Exocytosis, Genetic Structures, Reagents, Transfection, Targeted Control

\section{Introduction}

The increase in the life expectancy of the world's population, along with the success of healthcare, have led to an increase in the rate of neurodegenerative diseases, which are not yet curable. The most frequent neurodegenerative diseases include Parkinson's disease and, especially, Alzheimer's disease observed in more than $80 \%$ of patients of the older age groups suffering from dementia (Giau, 2015). According to official forecasts, by 2050 the number of Alzheimer's victims will triple from 47 million to 130 million patients (Thies and Bleiler, 2013). This fact actualizes the problem of searching for methods to prevent and treat epidemiologically significant neurodegenerative diseases.

One of the possible solutions of this problem is the development of personalized targeted therapy, qualitatively correcting errors of the genome. More and more research activity is devoted to such developments today (Nguyen et al., 2017). Unfortunately, no real breakthrough has been recorded in the world yet. However, given the speed of development of this direction, the emergence of know-how is quite possible. This is especially possible when combining efforts of specialists from various fields, including neurobiology, medicine and genetic engineering.

Currently, the vesicular hypothesis of the release of a neurotransmitters has been universally accepted, according to which the transfer of information from one brain cell to another occurs at the expense of a chemical agentneurotransmitter. Neurotransmitters are concentrated in vesicles and released from them through exocytosis. For this fact, the importance of astrocytes in brain function has been increasingly gathering an evidence in recent years. In addition to providing homeostatic control and metabolic support, they play an active role in processing information in neural circuits (Halassa and Haydon, 2010). As participants in two-way communication, astrocytes can both receive and send chemical signals to neurons. Studies have shown that astrocytes can respond to various neurotransmitters and factors and in turn release glutamate, D-serine, ATP and GammaAminobutyric Acid (GABA), as well as prostaglandins 
and neuropeptides in a process called gliothransmission

Various mechanisms of gliotransmission have been proposed, including $\mathrm{Ca}^{2+}$-controlled vesicular exocytosis and non-vesicular release. Non-vesicular release can be achieved through the ion channels of the plasma membrane, such as connexin/pannexin hemichannels, purinergic P2X7 channels, volume-regulated anion channels and pumps such as glutamate cysteine or glutamate uptake systems (Hamilton and Attwell, 2010). Until now, there is no clear evidence that channelmediated (connexin/pannexin/P2X7) mechanisms relate to physiological links between astrocytes and neurons, since opening of channels was recorded mainly under unusual physiological conditions such as trauma, convulsive activity and change in the concentration of oxygen and glucose (Giaume et al., 2013).

In this regard, $\mathrm{Ca}^{2+}$-dependent exocytosis has been proposed as the main mechanism for the release of main gliotransmitters (glutamate, D-serine and ATP), just as it occurs in neurons and secretory cells. An evidence that anionic channel of Bastropin-1 plays a role in the release of GABA and glutamate from astrocytes under unphysiological conditions has appeared (Lee et al., 2010). In addition, very fast (milliseconds), but $\mathrm{Ca}^{2+}$ independent release of glutamate was detected through TREK-1 potassium channel (Woo et al., 2012). However, it is still not clear how the channel that is structurally designed for selective release of a small cation (such as potassium) can adjust the release of negatively charged and larger organic anions such as glutamate. The question on whether the regulated exocytosis or channel-mediated mechanism (or both mechanisms) will be responsible for gliotransmission under physiological conditions is currently the subject of active discussion (Nedergaard and Verkhratsky, 2012). Neither the molecular mechanisms of gliotransmitters releasing nor their regulation have been fully studied. However, calcium is considered to be the main regulator of gliotransmission.

Due to the foregoing, it is very promising to study astroglia for targeting intracellular cascades and subsequent gene therapy.

The aim of this work is to create techniques based on genetic constructions to control exocytosis. This article presents the results of fragment of the project, consisting in testing experimental samples of the developed reagent kit for specific transfection of astroglial cells with the aim of targeted control of intracellular cascades in vivo and developing instructions for the use of this kit.

\section{Materials and Methods}

For the purpose of specific transfection of intracellular cascades in astrocytes in vitro, an experimental sample of the reagent kit was developed. It represented lentiviral genetic construction LVVGFAP-Case12 based on pTYF-SW-Linker shuttle vector
(Hamilton and Attwell, 2010; Zorec et al., 2012). in a DMEM culture media solution with $10 \%$ bovine serum (FS). The specificity of transfection of astroglial cells of the brain is provided by the presence of astrocyte-selective GFAP promoter (GFAP-Glial Fibrillar Acidic Protein). The possibility of targeted control of intracellular cascades is provided by the presence of the calcium indicator Case12.

Tests of the experimental sample of the reagent kit were carried out in vivo on acute brain slices of 17 adult Wistar rats. Transfection efficacy was assessed by counting the number of transfected cells on acute brain slices. We performed evaluation of the transfection efficiency on slices obtained from different regions of brain of the rat, since there is evidence of the regional heterogeneity of glial cells in the brain of mammals. Thus, for example an effective transfection of astrocytes from hippocampus does not mean that transfection of astrocytes of the brainstem will have the same effect. Selectivity of the transfection was checked by subsequent immunohistochemical staining of brain slices with polyclonal antibodies to astrocytic markers, such as GFAP and S100b. Effectiveness of the targeted control of intracellular cascades was assessed by the change in fluorescence intensity of Case 12 reporter protein in response to the addition of ATP in the solution.

Injection of virus particles into the brain was carried out according to the following protocol:

- We prepared working solutions: solution for brain preparation of the following composition $(\mathrm{mM}): \mathrm{NaCl}$ 87; $\mathrm{KCl} 2.5 ; \mathrm{MgSO}_{4}$ 8.48; $\mathrm{NaH}_{2} \mathrm{PO}_{4} 1.24 ; \mathrm{NaHCO}_{3}$ 26.2; $\mathrm{CaCl}_{2}$ 0.5; D-glucose 11; osmolarity (289-296 $\mathrm{mmol} / \mathrm{kg}$ ) and $\mathrm{pH}(7.4)$; solution for slices incubation of the following composition $(\mathrm{mM}): \mathrm{NaCl} 119 ; \mathrm{KCl}$ 2.5; $\mathrm{MgSO}_{4} 1.3 ; \mathrm{NaH}_{2} \mathrm{PO}_{4} 1 ; \mathrm{NaHCO}_{3} 26.2 ; \mathrm{CaCl}_{2} 1$; $\mathrm{MgCl}_{2} \quad 1.6$; D-glucose 11; osmolarity (289-296 $\mathrm{mmol} / \mathrm{kg}$ ) and $\mathrm{pH}$ (7.4). Ringer's working solution of the following composition (mM): $\mathrm{NaCl} 119 ; \mathrm{KCl} 2.5$; $\mathrm{MgSO}_{4} 1.3 ; \mathrm{NaH}_{2} \mathrm{PO}_{4} 1 ; \mathrm{NaHCO}_{3} 26.2 ; \mathrm{CaCl}_{2} 2$; Dglucose 11 (pH 7.4, osmolarity 295 mOsm)

- Rat was anesthetized by injection of zoletil and romitar, an animal was immobilized in stereotactic frame

- We made an opening for the cannula for injection, then the needle of the microdosage adapter was inserted into cannula and the solution was administered (LVV-GFAP-Case12 lentiviral genetic construction from the experimental reagent kit sample). Administration of virus particles was made slowly for $1 \mathrm{~min}$ with the volume of not more than $10 \mu \mathrm{L}$. Then the needle was removed from the cannula and the hole was closed. Postoperative care was provided to the operated animals

Tests of the experimental sample of the reagent kit for specific transfection of astroglial cells in vivo were performed according to the following protocol: 
- Decapitation of the rat was performed, for this skin and skull muscles were removed with the help of sharp-pointed straight scissors from the surface of the skull of the rat; then we made a transverse slice of the skull bones and then a longitudinal slice of the skull to the level of the eye sockets with the help of sharp-pointed straight scissors; then we made two transverse slices to the level of the eye sockets with the help of sharp-pointed straight scissors, after which the prepared skull bones were moved apart with forceps; cranial nerves were cut; isolated brain was immediately transferred to the preparation solution with subsequent cooling down to $+4^{\circ} \mathrm{C}$

- Isolated brain was adhered to the special magnetic substrate with vibratome 7000smz-2 (Campden Instruments LTD, England), the bath was filled with cooled solution for preparation and placed magnetic substrate with a brain into bath

- The prepared slices with the thickness of 40-50 $\mu \mathrm{m}$ were taken from the vibrotome bath with a brush and placed in a carbogenized solution for incubation with a temperature of $+36^{\circ} \mathrm{C}$

- Then a slice was placed under confocal microscope Zeiss 780 (Zeiss, Germany). For this purpose the brain slice in Ringer's solution was placed in a chamber with a certain gas and temperature regime, which was set by using Zen software (Carl Zeiss, Germany)

- Assessment of Case12 expression in astrocytes and monitoring of variations in the intensity of Case 12 fluorescence in response to change in intracellular calcium concentration in astrocytes were made by illumination (excitation) of the indicator with an argon laser with a wavelength of $488 \mathrm{~nm}$. This practically corresponds to th emaximum of Case12 excitation $(491 \mathrm{~nm})$. At the same time we used beam splitter MBS 458/543 or MBS 488/543/633 and the emission signal was detected in the spectrum $500-550 \mathrm{~nm}$

- Monitoring of the intensity of Case12 fluorescence in astrocytes was carried out in the time-series scanning mode. Before launching the experiment, we focused on the cut surface with an oil immersion $\mathrm{x} 40$ or $\mathrm{x} 63$ lens and ROI-regions or areas of interest were set, in which the change in fluorescence intensity in response to the stimulus would be measured

- In order to stimulate astrocytes, ATP in the final concentration of $50 \mu \mathrm{M}$ was added to the Ringer's solution; at the same time we continuously recorded an experiment in the Time series mode. Effectiveness of targeted control of intracellular cascades in astrocytes was assessed by the change in fluorescence intensity (an increase in fluorescence intensity of individual cells, marked as regions of interest - ROI, by 2 or more times)
- Acute slices after the experiment were fixed in $4 \%$ paraformaldehyde on phosphate buffer ( $\mathrm{pH}$ 7.4) for $2 \mathrm{~h}$ at the room temperature or $6 \mathrm{~h}$ at $+4^{\circ} \mathrm{C}$

- After fixation, the slices were washed in phosphate buffer at least three times for 15 min each and then incubated in $10 \%$ bovine serum solution for at least $6 \mathrm{~h}$ at $+4^{\circ} \mathrm{C}$

- After incubation with bovine serum, the slices were incubated for at least $24 \mathrm{~h}$ with primary antibodies to the astrocyte markers GFAP or S100b (1:1000)

- Slices were washed in phosphate buffer supplemented with $0.1 \%$ bovine serum and $0.1 \%$ Triton X detergent

- Slices were incubated with secondary antibodies conjugated with fluorescent labels for at least $6 \mathrm{~h}$ (1:1000)

- After incubation with secondary antibodies, the slices were washed in phosphate buffer at least three times for 15 min each, put into drug-filling medium containing DAPI DNA dye and examined under laser scanning confocal microscope LSM 780 (Carl Zeiss, Germany)

\section{Results}

Based on the results of tests of targeted control of intracellular cascades on 5 slices of the hippocampus and 5 slices of the brainstem in the rat, Regions of Interest (ROI) were isolated with cells expressing calcium indicator Case 12 and responding to ATP application by $\geq 2$-fold amplification of the fluorescence (Fig. 1 and 2).

Axis X labelled intensity of fluorescence in Relative Fluorescent Unit (RFU). Axis Y labelled absolute frequency i.e. number of point with certain fluorescence. Red, green and blue circles are the regions of interesting (ROI).

Fluorescence of Case 12 was characterized by one excitation peak $(\mathrm{Me}=491 \mathrm{~nm})$ and one emission peak $(516 \mathrm{~nm})$. In order to register fluorescent signal of Case12, we used the standard filter set for GFP (Green fluorescent protein). Calculations were carried out using the Origin software. The specificity of transfection of astroglial cells was checked by using immunohistochemical analysis. According to the data obtained, more than $90 \%$ of cells immunoreactive to GFAP were successfully transfected with the genetic construct LVV-GFAP-Case12 (Fig. 3 and 4).

Specificity of the transfection of astrocytes of hippocampus and brainstem with the genetic construction LVV-GFAP-Case 12 from the experimental sample of the reagent kit was comparable to the efficiency of transfection of astrocytes of the brainstem, besides no statistically significant differences were revealed (Fig. 5). 


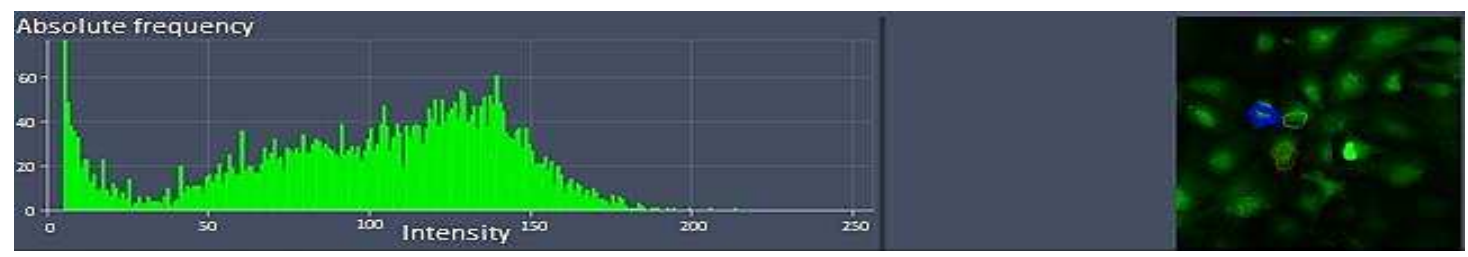

Fig. 1: Change in the fluorescence of calcium indicator Case12 in response to the addition of ATP in the final concentration of $50 \mu \mathrm{M}$ on the acute slices of the hippocampus (Mean Intensity 98.87, Standard Deviation 43.71, Pixels 4199; Area ( $\mu m$ x $\mu m)$ 723.6)

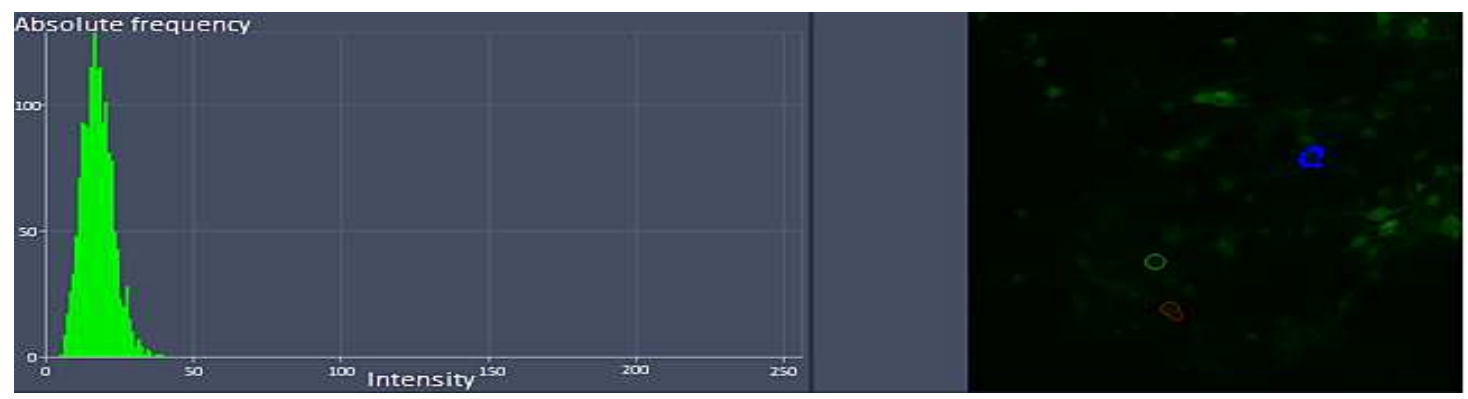

Fig. 2: Change in the fluorescence of calcium indicator Case12 in response to the addition of ATP in the final concentration of $50 \mu \mathrm{M}$ on the acute slices of the brainstem (Mean Intensity 17.14; Standard Deviation 5.19; Pixels 1520; Area ( $\mu m$ x $\mu m$ ) 261.95)
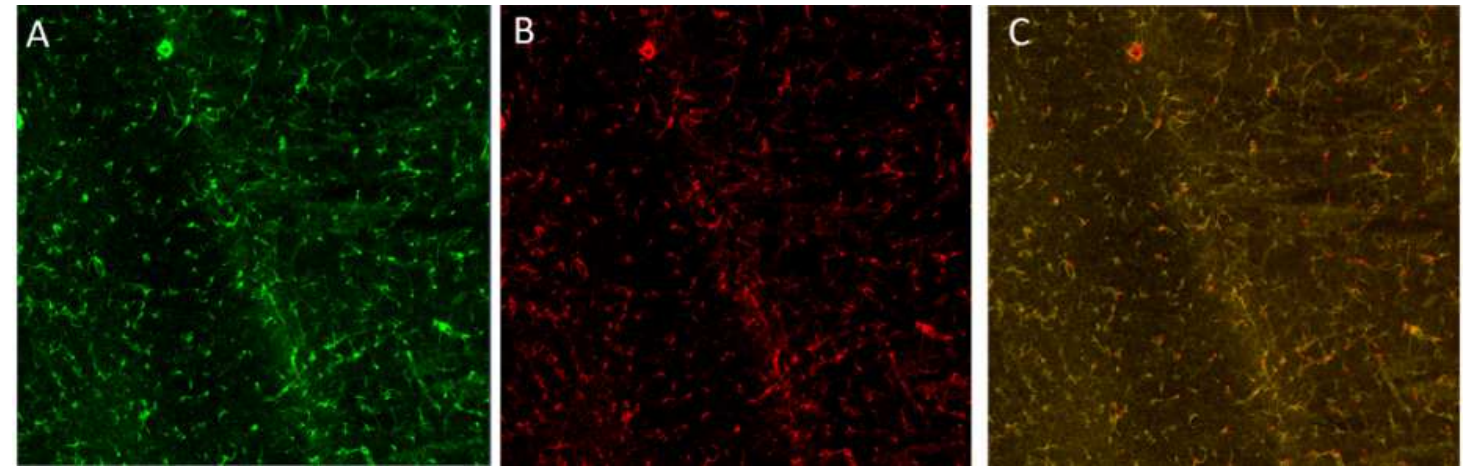

Fig. 3: Astrocytes from the acute hippocampal section transfected with calcium indicator Case12 (A, green) and immunohistochemical staining with anti-GFAP antibodies (B, red). colocalization of Case12 and GFAP staining (C, yellow)
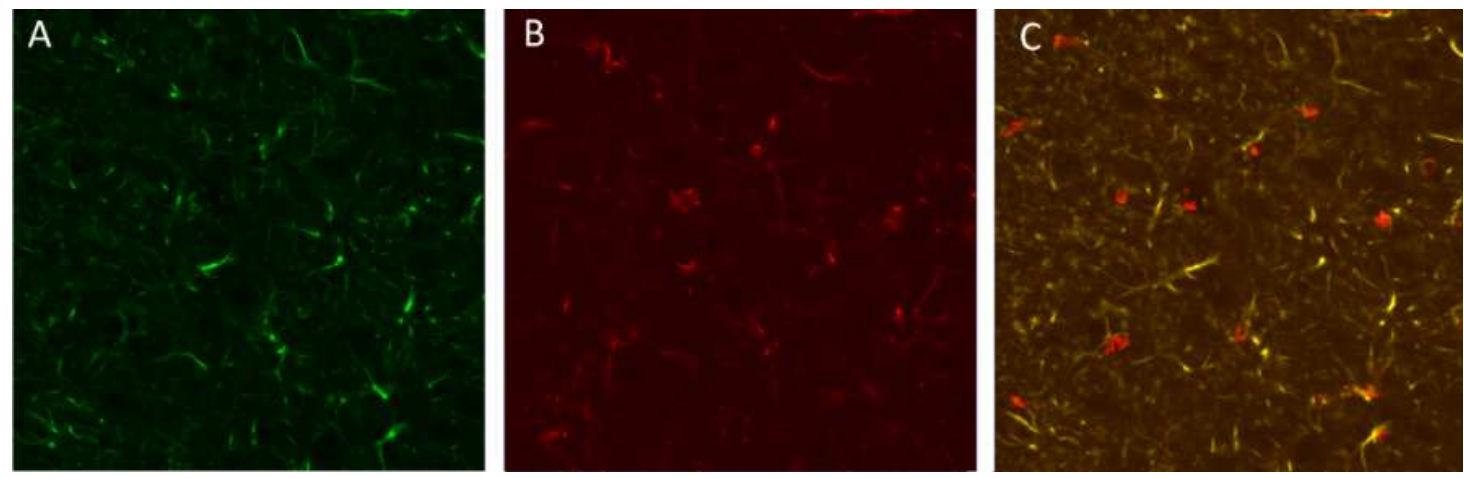

Fig. 4: Astrocytes from the acute brainstem section transfected with calcium indicator Case12 (A, green) and immunohistochemical staining with anti-GFAP antibodies (B, red). colocalization of Case12 and GFAP staining (C, yellow)

Thus, the experimental sample of the reagent kit for specific transfection of astroglial cells in vivo was successfully tested according to the above-described protocol. The use of the genetic construction LVVGFAP-Case12 developed from the experimental sample of the reagent kit from the experimental sample is an 
effective method of specific transfection of astroglial cells from different regions of the rat brain for targeted control of intracellular cascades in vivo.

In addition, instructions for the use of the reagent kit for specific transfection of astroglial cells have been developed with the aim of targeted control of intracellular cascades. The developed instructions for the use of the reagent kit for specific transfection of astroglial cells with the aim of targeted control of intracellular cascades are also based on the experimental data obtained by testing experimental samples of the reagent kit for specific transfection of astroglial cells with the aim of targeted control of intracellular cascades.
The $72 \mathrm{~h}$ analysis of the sensitivity of the cell line to transfection with lentiviruses by using the control line of astrocyte culture in order to select an optimal titre of lentiviral particles demonstrated safety of the technology for different parts of the brain.

In order to obtain stable cell line with an effective level of expression of Case12 calcium indicator, $10 \mu \mathrm{L}$ of lentiviral particles were used and the growth medium was changed every $24 \mathrm{~h}$. We revealed a directly proportional increase in the number of cells successfully transfected with LVV-GFAP-Case12 lentiviral construction in both hippocampus and the brainstem (Fig. 6).

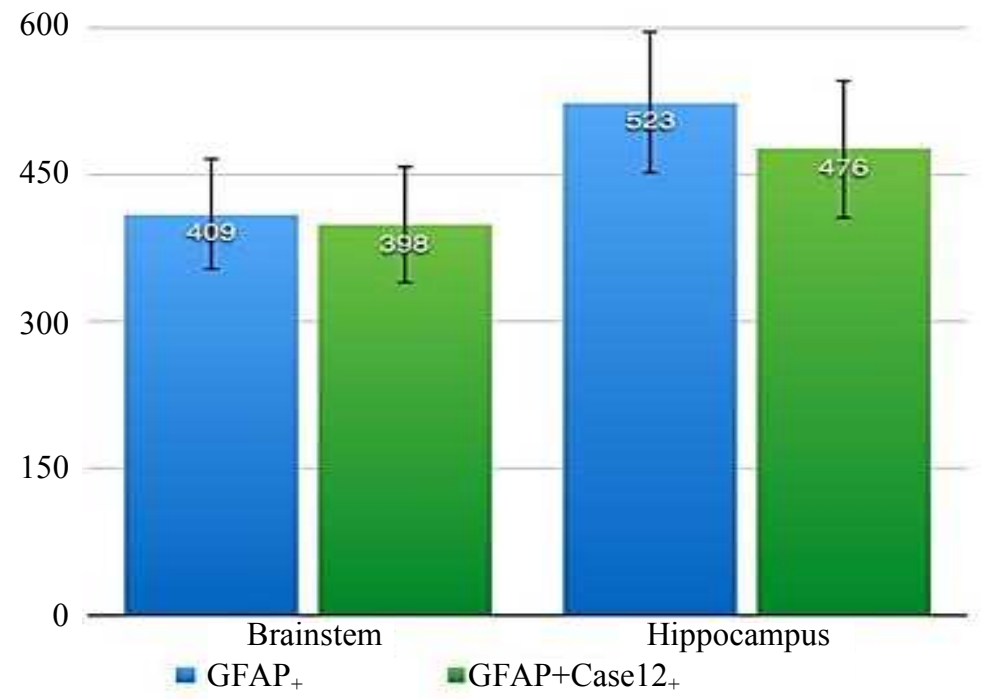

Fig. 5: Number of astrocytes of brainstem and hippocampus transfected with the genetic construction LVV-GFAP-Case12 $(\mathrm{GFAP}+\mathrm{Case} 12+)$, to the total number of astrocytes immunoreactive to GFAP $(\mathrm{GFAP}+)$. The data are presented as mean values of the number of cells \pm SEM. The data are obtained after $72 \mathrm{~h}$ from the moment of transfection

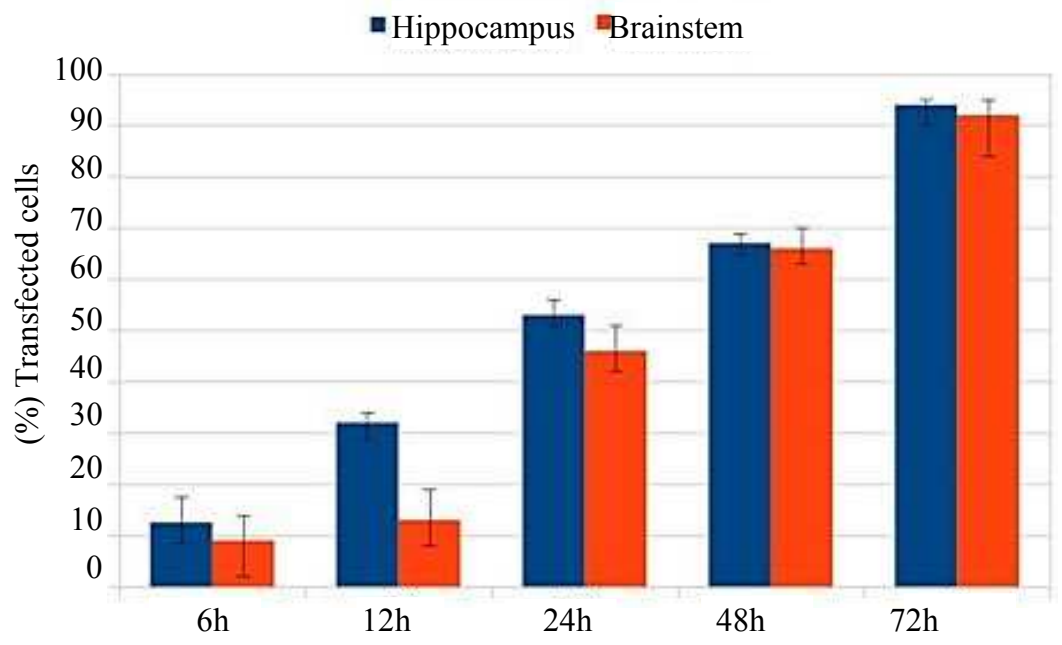

Fig. 6: Number of cells successfully transfected with lentiviral construction LVV-GFAP-Case12 


\section{Discussion}

The result of this study relates to the field of cellular and molecular biology, in particular, to lentiviral reporter vector systems for the analysis of intracellular signaling cascades in mammalian brain cells. Despite the widespread use of genetic constructions based on adenoviruses and adeno-associated viruses, lentiviral constructions have one important advantage: they successfully transfuse both dividing and non-dividing cells (after mitosis), which is a key factor for stable and longterm expression of the target gene in brain cells. At the present time chemo- and optogenetic methods are widely used in neurobiological studies. Genetic constructions that allow an insertion of a specific gene into target cells, both in vitro and in vivo, represent the high-tech and precise method that allows solving a wide variety of neurobiological problems. However, each method has its advantages and disadvantages. One of limitations of working with virus-based vector systems is the lack of selectivity of cell transfection, or low efficiency of transfection of the analyzed cells. The genetic construction developed by us should provide highly efficient and highly selective transfection of targeted cells (cell populations) and stable expression of the gene.

The reagent kit created within the framework of this project allows successfully (i.e. efficiently and selectively) transfect astroglial cells of the mammalian brain with the purpose of subsequent monitoring of calcium dynamics. This allows to solve one of the most urgent problems of modern neuroscience methodically - to transfect glial cells of the brain with the aim of targeted control of intracellular cascades. Targeted control of intracellular calciumdependent signaling cascades in astrocytes by using a developed reagent kit will be used in further studies of the mammalian brain functioning, as well as in the development of new methods for targeted manipulation of brain cells, including glial cells and neurons. Glia, unlike neurons, remains largely unexplored. That is why glial cells are the subject of many advanced studies. The necessity for molecular tools and new methods of research and manipulation of intracellular signaling cascades, as well as mechanisms of intercellular communication, is constantly growing. Thus, the genetic construction developed within the framework of this project as part of the reagent kit will find wide application in further fundamental scientific and applied research studies, as well as in drug testing and treatment effectiveness in future studies.

\section{Conclusion}

Thus, the obtained experimental results allow us to say that the use of the developed genetic construction LVV-GFAP-Case12 is an effective method of specific transfection of astroglial cells from different regions of the rat brain for targeted control of intracellular cascades. The developed and tested instructions on the use of the reagent kit for specific transfection of astroglial cells with the aim of targeted control of intracellular cascades allow the next step in the implementation of the project for the development of genetic structures for exocytosis control, which in turn will help to ensure the success of targeted therapy for various neurological diseases, including neurodegenerative ones.

\section{Acknowledgement}

This work was supported by the grant from the Ministry of Education and Science of the Russian Federation (FCP No. 14.575.21.0036 (unique identificator-RFMEFI57514X0036) at the Immanuel Kant Baltic Federal University.

\section{Author's Contributions}

All authors contributed equally.

\section{Ethics}

This article is original and contains unpublished material. The corresponding author confirms that all of the other authors have read and approved the manuscript and there are no ethical issues involved.

\section{References}

Giau, V.V., S.S. An, E. Bagyinszky and S.Y. Kim, 2015. Next Generation Sequencing (NGS) Gene panels and primers for studies on neurodegenerative disorders. Toxicol. Environ. Health Sci., 7: S32- S32.

Giaume, C., L. Leybaert, C.C. Naus and J.C. Saez, 2013. Connexin and pannexin hemichannels in brain glial cells: Properties, pharmacology and roles. Frontiers Pharmacol.

DOI: 10.3389/fphar.2013.00088

Halassa, M.M. and P.G. Haydon, 2010. Integrated brain circuits: Astrocytic networks modulate neuronal activity and behavior. Ann. Rev. Physiol., 72: 335-335.

Hamilton, N.B. and D. Attwell, 2010. Do astrocytes really exocytose neurotransmitters? Nature Rev. Neurosci., 11: 227-238. DOI: 10.1038/nrn2803

Lee, S., B.E. Yoon, K. Berglund, S.J. Oh and H. Park et al., 2010. Channel-mediated tonic GABA release from Glia. Science, 330: 790-796.

DOI: $10.1126 /$ science. 1184334

Nedergaard, M. and A. Verkhratsky, 2012. Artifact versus reality - how astrocytes contribute to synaptic events. Glia, 60: 1013-1023.

Nguyen, T.T., V.V. Giau and T.K. Vo, 2017. Current advances in transdermal delivery of drugs for Alzheimer's disease. Indian J. Pharmacol., 49: 145-154.

DOI: $10.4103 / 0253-7613.208143$ 
Thies, W. and L. Bleiler, 2013. Alzheimer's disease: Facts and figures. Alzheimers Dement, 9: 208-215.

Woo, D.H., K.S. Han, J.W. Shim, B.E. Yoon and E. Kim et al. 2012. TREK-1 and Best1 channels mediate fast and slow glutamate release in astrocytes upon GPCR activation. Cell, 151: 25-40. DOI: $10.1016 /$ j.cell.2012.09.005
Zorec, R., A. Araque and G. Carmignoto, 2012. Astroglial excitability and gliotransmission: An appraisal of $\mathrm{Ca}^{2+}$ as a signalling route. ASN Neuro, 4: e00075-e00075.

DOI: $10.1042 / A N 20110061$ 